\title{
OA03 I-03. Increased regulatory T cell frequency and HIV-I specific suppression after therapeutic vaccination of HIV-infected patients on antiretroviral therapy
} BC Macatangay*1, ME Szajnik ${ }^{2}$, TL Whiteside ${ }^{2}$, SA Riddler ${ }^{1}$ and CR Rinaldo ${ }^{3}$

Address: ${ }^{1}$ Division of Infectious Diseases, University of Pittsburgh, Pittsburgh, PA, USA, ${ }^{2}$ University of Pittsburgh Hillman Cancer Center, Pittsburgh, PA, USA and ${ }^{3}$ University of Pittsburgh Graduate School of Public Health, Pittsburgh, PA, USA

* Corresponding author

from AIDS Vaccine 2009

Paris, France. 19-22 October 2009

Published: 22 October 2009

Retrovirology 2009, 6(Suppl 3):O2I doi:I0.II86/I742-4690-6-S3-O2I

This abstract is available from: http://www.retrovirology.com/content/6/S3/O2I

(c) 2009 Macatangay et al; licensee BioMed Central Ltd.

\section{Background}

We tested the hypothesis that therapeutic vaccination against HIV-1 can lead to an increase in the frequency and suppressive function of regulatory, CD4+ T cells (Treg).

\section{Methods}

HIV-1(+) subjects on ART $(\mathrm{n}=17)$ were enrolled in a phase I therapeutic vaccine trial where they received 2 doses of autologous dendritic cells loaded with HIV-1 peptides. Peripheral blood mononuclear cells (PBMC) obtained from the subjects pre- and post-vaccine, and from normal controls (NC) were stained with antibodies specific for Treg (CD4+CD25hiFOXP3+), CD45RO, GITR, and CTLA4 and assayed by flow cytometry. PBMC pre and post-vaccine from 7 subjects were also evaluated for polyfunctionality using a flow cytometry-based, CD8+ T cell intracellular cytokine staining assay for 5 immune mediators after stimulation with Gag peptide, staphylococcal enterotoxin B (SEB) and medium alone. Treg were depleted in one set, and total vaccine response (post-vaccine - pre-vaccine) was compared in the Treg(+) and Tregdepleted (Treg-) sets.

\section{Results}

After vaccination, 12/17 subjects had increased Treg frequency from $0.74 \%$ to $1.2 \%(\mathrm{p}=0.06)$; the median increase was $30 \%$. Of the 11 patients whose CD $8+T$ cells did not respond to the vaccine by an increase in production of interferon $\gamma$ (ELISPOT assay), 7 (64\%) had increased frequencies of Treg. Although there was no significant change in CD8 + T cell polyfunctionality after vaccination, depletion of Treg resulted in increased polyfunctionality post-vaccine ( $\mathrm{p}=0.029)$, with the percentage of $\mathrm{CD} 8+\mathrm{T}$ cells producing more than 1 immune mediator increasing to more than twice the pre-vaccine levels. There was no difference in polyfunctionality in the Treg(+) and Treg(-) sets when stimulated with SEB, implying specificity of suppression to HIV-1 antigens.

\section{Conclusion}

Therapeutic immunization against HIV-1 causes a modest increase in Treg frequency and a significant increase in HIV-1-specific, Treg suppressive function. The role of Treg should be considered in immunotherapeutic trials of HIV1 infection. 